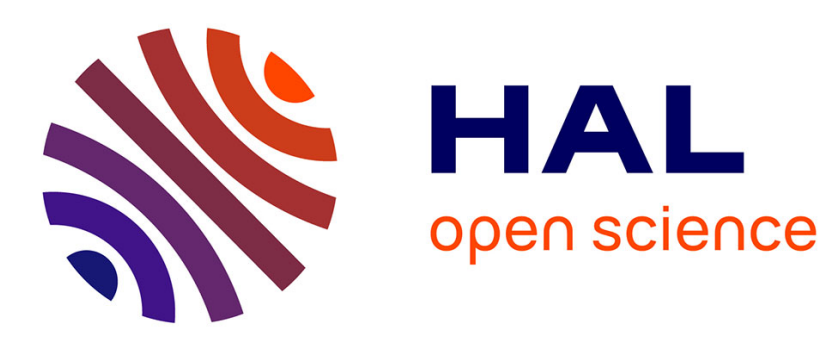

\title{
Characterizations of bivariate models using dynamic Kullbak-Leibler discrimination measures
}

\author{
J. Navarro, S.M. Sunoj, M.N. Linu
}

\section{To cite this version:}

J. Navarro, S.M. Sunoj, M.N. Linu. Characterizations of bivariate models using dynamic KullbakLeibler discrimination measures. Statistics and Probability Letters, 2011, 81 (11), pp.1594. 10.1016/j.spl.2011.05.016 . hal-00789866

\section{HAL Id: hal-00789866 https://hal.science/hal-00789866}

Submitted on 19 Feb 2013

HAL is a multi-disciplinary open access archive for the deposit and dissemination of scientific research documents, whether they are published or not. The documents may come from teaching and research institutions in France or abroad, or from public or private research centers.
L'archive ouverte pluridisciplinaire HAL, est destinée au dépôt et à la diffusion de documents scientifiques de niveau recherche, publiés ou non, émanant des établissements d'enseignement et de recherche français ou étrangers, des laboratoires publics ou privés. 


\section{Accepted Manuscript}

Characterizations of bivariate models using dynamic Kullbak-Leibler

discrimination measures

J. Navarro, S.M. Sunoj, M.N. Linu

PII:

S0167-7152(11)00191-X

DOI: $\quad$ 10.1016/j.spl.2011.05.016

Reference: STAPRO 6013

To appear in: Statistics and Probability Letters

Received date: 11 February 2011

Revised date: 9 May 2011

Accepted date: 26 May 2011

Please cite this article as: Navarro, J., Sunoj, S.M., Linu, M.N., Characterizations of bivariate models using dynamic Kullbak-Leibler discrimination measures. Statistics and Probability Letters (2011), doi:10.1016/j.spl.2011.05.016

This is a PDF file of an unedited manuscript that has been accepted for publication. As a service to our customers we are providing this early version of the manuscript. The manuscript will undergo copyediting, typesetting, and review of the resulting proof before it is published in its final form. Please note that during the production process errors may be discovered which could affect the content, and all legal disclaimers that apply to the journal pertain. 


\title{
Characterizations of bivariate models using dynamic Kullbak-Leibler discrimination measures
}

\author{
J. Navarro ${ }^{1, a}$, S.M. Sunoj ${ }^{b}$, M.N. Linu ${ }^{\mathrm{b}}$ \\ ${ }^{a}$ Universidad de Murcia, Murcia, Spain \\ ${ }^{b}$ Cochin University of Science and Technology, Cochin, Kerala, India
}

\begin{abstract}
In this paper the residual Kullback-Leibler discrimination information measure is extended to conditionally specified models. The extension is used to characterize some bivariate distributions. These distributions are also characterized in terms of proportional hazard rate models and weighted distributions. Moreover, we also obtain some bounds for this dynamic discrimination function by using the likelihood ratio order and some preceding results.
\end{abstract}

Keywords: Kullback-Leibler discrimination information, weighted distributions, conditionally specified models, proportional hazard rate. 2000 MSC: 62N05, 62B10

\section{Introduction}

Let $X$ and $Y$ be two absolutely continuous random variables with the common support $S=(l, \infty)$ for $l \geq 0$. Denote by $f, F$ and $\bar{F}$, the probability

\footnotetext{
${ }^{1}$ Corresponding author.

Address: Facultad de Matematicas, Universidad de Murcia, 30100 Murcia, Spain. E-mail: jorgenav@um.es Tel.: 34868883509 Fax.: 34868884182
} 
density function (PDF), the cumulative distribution function (CDF) and the survival (or reliability) function (SF) of $X$, respectively, and by $g, G$ and $\bar{G}$, the corresponding functions of $Y$. As an information distance between $X$ and $Y$, Kullback and Leibler (1951) proposed a directed divergence (also known as information divergence, information gain, relative entropy or discrimination measure) given by

$$
I_{X, Y}=\int_{l}^{\infty} f(x) \log \frac{f(x)}{g(x)} d x .
$$

This function is a measure of the similarity (closeness) between the two distributions and it plays an important role in information theory, reliability and other related fields. Further, note that if $f=g$ (a.e.), then $I_{X, Y}=0$.

Length of time during a study period has been considered as a prime variable of interest in many fields such as reliability, survival analysis, economics, business, etc. In particular, consider an item under study, then the information about the residual (or past) lifetime is an important task in many applications. In such cases, the information measures are functions of time, and thus they are dynamic. Based on this idea, Ebrahimi and Kirmani (1996) defined the Kullback-Leibler discrimination information measure of $X$ and $Y$ at time $t$ by

$$
I_{X, Y}(t)=\int_{t}^{\infty} \frac{f(x)}{\bar{F}(t)} \log \frac{f(x) \bar{G}(t)}{g(x) \bar{F}(t)} d x .
$$

Note that $I_{X, Y}(t)=I_{X_{t}, Y_{t}}$, where $X_{t}=(X-t \mid X>t)$ and $Y_{t}=(Y-t \mid Y>$ t) are the residual lifetimes associated to $X$ and $Y$. A similar function is obtained in terms of the inactivity times $(t-X \mid X<t)$ and $(t-Y \mid Y<t)$ in Di Crescenzo and Longobardi (2004) (see also Maya and Sunoj, 2008). Interesting extensions to the multivariate case are obtained in Ebrahimi et 
al. (2007). For additional information on these measures see Ebrahimi et al. (2010) and the references therein.

The concept of weighted distributions is usually considered in connection with modeling statistical data, where the usual practice of employing standard distributions is not found appropriate in some cases. In recent years, this concept has been applied in many areas of statistics, such as analysis of family size, human heredity, wildlife population study, renewal theory, biomedical, statistical ecology, reliability modeling, etc. Associated to a random variable $X$ with $\operatorname{PDF} f$ and to a nonnegative real function $w$, we can define the weighted random variable $X^{w}$ with density function

$$
f^{w}(x)=\frac{w(x) f(x)}{E(w(X))}
$$

where we assume $0<E(w(X))<\infty$. When $w(x)=x, X^{w}$ is called the length (or size) biased random variable and it is denoted by $X^{*}$. For recent works on weighted distributions, we refer the reader to Bartoszewicz and Skolimowska (2006); Navarro et al. (2006); Blazej (2008); Maya and Sunoj (2008); Bartoszewicz (2009); Navarro and Sarabia (2010); Sunoj and Linu (2011).

The obtention of the joint distribution of $(X, Y)$ when conditional distributions of $(X \mid Y=y)$ and $(Y \mid X=x)$ are known has been an important problem dealt with by many researchers in the past. This approach of identifying a bivariate density using the conditionals is called the conditional specification of the joint distribution (see Arnold et al., 1999). These conditional models are often useful in many two component reliability systems when the operational status of one component is known.

In the present paper, the Kullback-Leibler discrimination information 
measure $I_{X, Y}(t)$ proposed by Ebrahimi and Kirmani (1996) is extended to conditionally specified models. This extension is used to characterize some bivariate distributions. These distributions are also characterized in terms of proportional hazard rate models and weighted distributions. These results are given in Section 2. Moreover, in Section 3, we obtain bounds for this dynamic discrimination function by using the likelihood ratio order and some preceding results. The proof of the main result is given in Section 4 .

\section{Main results}

Let $\left(X_{1}, X_{2}\right)$ and $\left(Y_{1}, Y_{2}\right)$ be two bivariate random vectors with joint PDF $f$ and $g$, joint $\operatorname{CDF} F$ and $G$ and joint $\operatorname{SF} \bar{F}$ and $\bar{G}$, respectively. Let us assume that the common support is $S=(l, \infty) \times(l, \infty)$ for $l \geq 0$. Also let $f_{i}(s \mid t)$ and $g_{i}(s \mid t), \bar{F}_{i}(s \mid t)$ and $\bar{G}_{i}(s \mid t)$ denote the PDF and the SF of $\left(X_{i} \mid X_{3-i}=t\right)$ and $\left(Y_{i} \mid Y_{3-i}=t\right)$, respectively, for $i=1,2$. Then we define the conditional Kullback-Leibler discrimination (CKLD) information functions as

$$
I_{X_{i}, Y_{i}}(s \mid t)=\int_{s}^{\infty} \frac{f_{i}(x \mid t)}{\bar{F}_{i}(s \mid t)} \log \frac{f_{i}(x \mid t) \bar{G}_{i}(s \mid t)}{g_{i}(x \mid t) \bar{F}_{i}(s \mid t)} d x
$$

for $i=1,2$ and $s, t \geq l$. Note that

$$
I_{X_{i}, Y_{i}}(s \mid t)=I_{\left(X_{i} \mid X_{3-i}=t\right),\left(Y_{i} \mid Y_{3-i}=t\right)}(s)
$$

for $i=1,2$ and $s, t \geq l$. Hence $I_{X_{i}, Y_{i}}(s \mid t)$ is the dynamic Kullback-Leibler discrimination information measure at time $s$ defined by Ebrahimi and Kirmani (1996) but applied to the conditional random variables $\left(X_{i} \mid X_{3-i}=t\right)$ and $\left(Y_{i} \mid Y_{3-i}=t\right)$ for $i=1,2$. As in the univariate case these functions 
measure the information distance between the residual lifetimes of the conditional distributions of the two random vectors. Of course, in the bivariate case there are other interesting options (see, e.g., Ebrahimi et al., 2007).

In survival studies, the most widely used semi-parametric model is the proportional hazard rate (PHR) Cox model. Let $X$ and $Y$ be two random variables with the same support $S$ and with hazard rate functions $h_{X}=f / \bar{F}$ and $h_{Y}=g / \bar{G}$, respectively. Then $X$ and $Y$ satisfy the PHR model when

$$
h_{Y}(t)=\theta h_{X}(t)
$$

for all $t \in S$. This relationship is also equivalent to

$$
\bar{G}(t)=(\bar{F}(t))^{\theta}
$$

for all $t$ (see Cox, 1959). Ebrahimi and Kirmani (1996) obtained the following result.

Theorem 1 (Ebrahimi and Kirmani (1996)). The function $I_{X, Y}(t)$ is constant if and only if $X$ and $Y$ satisfy the PHR model.

In a similar way the random vectors $\left(X_{1}, X_{2}\right)$ and $\left(Y_{1}, Y_{2}\right)$ satisfy the conditional proportional hazard rate (CPHR) model (see Sankaran and Sreeja, 2007) when their respective conditional hazard rate functions satisfy

$$
h_{\left(Y_{i} \mid Y_{3-i}\right)}(s \mid t)=\theta_{i}(t) h_{\left(X_{i} \mid X_{3-i}\right)}(s \mid t)
$$

for $i=1,2$, where $\theta_{i}(t)$ is a nonnegative function of $t$. Then we can state the result as follows.

Theorem 2. For $i=1,2$, the function $I_{X_{i}, Y_{i}}(s \mid t)$ only depends on $t$ if and only if $\left(Y_{i} \mid Y_{3-i}=t\right)$ and $\left(X_{i} \mid X_{3-i}=t\right)$ satisfy the CPHR model (2). 
The proof is obtained from Theorem 1 and (1).

Next let us consider the random vector $\left(X_{1}^{w}, X_{2}^{w}\right)$ which has the bivariate weighted distribution associated to $\left(X_{1}, X_{2}\right)$ and to two nonnegative real functions $w_{1}$ and $w_{2}$, that is, its joint $\mathrm{PDF}$ is

$$
f^{w}\left(x_{1}, x_{2}\right)=\frac{w_{1}\left(x_{1}\right) w_{2}\left(x_{2}\right) f\left(x_{1}, x_{2}\right)}{E\left(w_{1}\left(X_{1}\right) w_{2}\left(X_{2}\right)\right)} .
$$

It is easy to see that the marginal random variable $X_{i}^{w}$ has the (univariate) weighted distribution associated to $X_{i}$ and $X_{i}$ for $i=1,2$. In particular, the length biased bivariate random vector, denoted by $\left(X_{1}^{*}, X_{2}^{*}\right)$, is obtained when $w_{1}(x)=w_{2}(x)=x$. There are other options in defining the bivariate weighted distribution which can be found in Navarro et al. (2006).

Now we can state the main result of the paper as follows.

Theorem 3. Let $\left(X_{1}^{w}, X_{2}^{w}\right)$ be a random vector which has the bivariate weighted distribution associated to $\left(X_{1}, X_{2}\right)$ and to two nonnegative and differentiable functions $w_{1}$ and $w_{2}$. Let us assume that the support of $\left(X_{1}, X_{2}\right)$ is $S=(l, \infty) \times(l, \infty)$ for $l \geq 0$. Then the following conditions are equivalent:

(a) $\left(X_{1}^{w}, X_{2}^{w}\right)$ and $\left(X_{1}, X_{2}\right)$ satisfy the CPHR model (2) for $i=1,2$.

(b) $I_{X_{i}, X_{i}^{w}}(s \mid t)$ only depends on $t$ for $i=1,2$.

(c) The conditional reliability functions of $\left(X_{1}, X_{2}\right)$ satisfy

$$
\log \bar{F}_{i}(s \mid t)=\frac{\log \left(w_{i}(s) / w_{i}(l)\right)}{\theta_{1}(t)-1}
$$

for $i=1,2$.

(d) $\left(X_{1}, X_{2}\right)$ has the following joint PDF

$f\left(x_{1}, x_{2}\right)=c a_{1} a_{2} \frac{w_{1}^{\prime}\left(x_{1}\right) w_{2}^{\prime}\left(x_{2}\right)}{w_{1}^{a_{1}+1}\left(x_{1}\right) w_{2}^{a_{2}+1}\left(x_{2}\right)} \exp \left(-\phi a_{1} a_{2}\left(\log \frac{w_{1}\left(x_{1}\right)}{w_{1}(l)}\right)\left(\log \frac{w_{2}\left(x_{2}\right)}{w_{2}(l)}\right)\right)$ for $x_{1}, x_{2} \geq l$, where $c>0, \phi \geq 0$ and $a_{i}>1$ or $a_{i}<0$ for $i=1,2$. 
The proof is given in Section 4. Note that the conditions given in Theorem 3, (c), imply that either $\log \left(w_{i}(s) / w_{i}(l)\right)$ or $-\log \left(w_{i}(s) / w_{i}(l)\right)$ should be cumulative hazard rate functions, that is, they should be nonnegative, increasing and they should go to $\infty$ when $s$ goes to $\infty$. In the first case, $w_{i}$ should be increasing in $[l, \infty)$ with $w_{i}(l)>0$ and $w_{i}(\infty)=\infty$. In the second case, $w_{i}$ should be decreasing, with $w_{i}(s)>0$ for $s \in[l, \infty)$ and $w_{i}(\infty)=0$. These conditions can also be written as

$$
h_{i}(s \mid t)=\frac{w_{i}^{\prime}(s) / w_{i}(s)}{1-\theta_{i}(t)}
$$

for $i=1,2$, that is, $\left(X_{1} \mid X_{2}=t\right)$ and $\left(X_{2} \mid X_{1}=t\right)$ satisfy the conditional proportional hazard rate model considered by Arnold and Strauss (1988). The reliability properties of this semiparametric model can be seen in Navarro and Sarabia (2011). Actually, the model in (d) is just a truncated version of Arnold and Strauss model in the support $S=(l, \infty) \times(l, \infty)$ and when $l=0$ both models coincide. Again we have two options, in the first one, $\lambda_{i}(s)=w_{i}^{\prime}(s) / w_{i}(s)$ is a proper hazard rate function and, in the second one, $\lambda_{i}(s)=-w_{i}^{\prime}(s) / w_{i}(s)$ is a proper hazard rate function. In the first option, we need $a_{i}>1$ and in the second one $a_{i}<0$, for $i=1,2$. The model in (d) contains several parametric models. In particular, when $l=1$ and $w_{1}(x)=w_{2}(x)=x$ for $x>1$, from Theorem 3 , we can characterize the bivariate Pareto model with the following joint PDF

$$
f\left(x_{1}, x_{2}\right)=\frac{c a_{1} a_{2}}{x_{1}^{a_{1}+1} x_{2}^{a_{2}+1}} \exp \left(-\phi a_{1} a_{2}\left(\log x_{1}\right)\left(\log x_{2}\right)\right)
$$

for $x_{1}, x_{2} \geq 1$, where $c>0, a_{1}, a_{2}>1$ and $\phi \geq 0$. 


\section{Bounds}

In this section we obtain bounds for the CKLD functions by using the likelihood ratio (LR) order. The results are similar to that given in Di Crescenzo and Longobardi (2004). First we need the definition of the LR order. If $X$ and $Y$ have PDF $f$ and $g$, respectively, then $X$ is said to be less than $Y$ in the likelihood ratio order (denoted by $X \leq_{L R} Y$ ) if $g / f$ is increasing in the union of their supports. Then we have the following results.

Theorem 4. For $i=1,2$, if $\left(X_{i} \mid X_{3-i}=t\right) \leq_{L R}\left(Y_{i} \mid Y_{3-i}=t\right)$, then

$$
I_{X_{i}, Y_{i}}(s \mid t) \leq \log \frac{h_{X_{i} \mid X_{3-i}}(s \mid t)}{h_{Y_{i} \mid Y_{3-i}}(s \mid t)}
$$

Theorem 5. For $i=1,2$, if $w_{i}$ is increasing, then

$$
I_{X_{i}, X_{i}^{w}}(s \mid t) \leq \log \frac{E\left(w_{i}\left(X_{i}\right) \mid X_{i}>s, X_{3-i}=t\right)}{w_{i}(s)} .
$$

Theorem 6. For $i=1,2$, if $\left(Y_{i} \mid Y_{3-i}=t\right) \leq_{L R}\left(Z_{i} \mid Z_{3-i}=t\right)$, then

$$
I_{X_{i}, Y_{i}}(s \mid t) \geq I_{X_{1}, Z_{1}}(s \mid t)+\frac{h_{Z_{i} \mid Z_{3-i}}(s \mid t)}{h_{Y_{1} \mid Y_{2}}(s \mid t)} .
$$

\section{Proof of Theorem 3}

The equivalence between (a) and (b) is a consequence of Theorem 2 .

Let us prove that (a) implies (c). So let us assume that $\left(X_{1}^{w}, X_{2}^{w}\right)$ and $\left(X_{1}, X_{2}\right)$ satisfy the CPHR model (2) for $i=1,2$. From the expression of the PDF of $\left(X_{1}^{w}, X_{2}^{w}\right)$ given in (3), it is easy to prove that the PDF of $\left(X_{i}^{w} \mid X_{3-i}^{w}=t\right)$ is given by

$$
f_{i}^{w}(s \mid t)=\frac{w_{i}(s) f_{i}(s \mid t)}{E\left(w_{i}\left(X_{i}\right) \mid X_{3-i}=t\right)}
$$


for $i=1,2$, where $f_{i}(s \mid t)$ is the PDF of $\left(X_{i} \mid X_{3-i}=t\right)$. Then, for $i=1,2$, the hazard rate $h_{i}^{w}(s \mid t)$ of $\left(X_{i}^{w} \mid X_{3-i}^{w}=t\right)$ is given by

$$
h_{i}^{w}(s \mid t)=\frac{w_{i}(s) f_{i}(s \mid t)}{\int_{s}^{\infty} w_{i}(x) f_{i}(x \mid t) d x} .
$$

Moreover, from (2), we have

$$
h_{i}^{w}(s \mid t)=\theta_{i}(t) h_{i}(s \mid t)
$$

and hence

$$
\frac{w_{i}(s) f_{i}(s \mid t)}{\int_{s}^{\infty} w_{i}(x) f_{i}(x \mid t) d x}=\theta_{i}(t) \frac{f_{i}(s \mid t)}{\bar{F}_{i}(s \mid t)}
$$

Therefore,

$$
\frac{1}{\bar{F}_{i}(s \mid t)} \int_{s}^{\infty} w_{i}(x) f_{i}(x \mid t) d x=\frac{1}{\theta_{i}(t)} w_{i}(s) .
$$

Then, differentiating both sides with respect to $s$, we obtain

$$
-w_{i}(s) f_{i}(s \mid t)=\frac{1}{\theta_{i}(t)}\left(w_{i}^{\prime}(s) \bar{F}_{i}(s \mid t)-w_{i}(s) f_{i}(s \mid t)\right),
$$

that is,

$$
h_{i}(s \mid t)=\frac{w_{i}^{\prime}(s)}{\left(1-\theta_{i}(t)\right) w_{i}(s)}
$$

Hence

$$
\log \bar{F}_{i}(s \mid t)=-\int_{l}^{s} h_{i}(x \mid t) d x=\frac{1}{\left(\theta_{i}(t)-1\right)} \log \frac{w_{i}(s)}{w_{i}(l)}
$$

for $i=1,2$ and (c) holds.

Let us prove that (c) is equivalent to $(\mathrm{d})$. The expressions given in (c) are equivalent to

$$
h_{i}(s \mid t)=\frac{w_{i}^{\prime}(s) / w_{i}(s)}{1-\theta_{i}(t)}
$$


for $i=1,2$, that is, $\left(X_{1} \mid X_{2}=t\right)$ and $\left(X_{2} \mid X_{1}=t\right)$ satisfy the conditional proportional hazard rate model considered by Arnold and Strauss (1988) which is equivalent to $(\mathrm{d})$.

Finally, let us prove that (d) implies (a). From the expression of the joint PDF given in (d) it is easy to prove that the conditional hazard rate functions are given by

$$
h_{i}(s \mid t)=a_{i}\left(1-\phi a_{3-i} \log \frac{w_{3-i}(t)}{w_{i}(l)}\right) \frac{w_{i}^{\prime}(s)}{w_{i}(s)}
$$

for $i=1,2$. Moreover, the weighted version associated to $w_{1}$ and $w_{2}$ has the following joint PDF

$$
f^{w}\left(x_{1}, x_{2}\right)=c \frac{w_{1}^{\prime}\left(x_{1}\right) w_{2}^{\prime}\left(x_{2}\right)}{w_{1}^{a_{1}}\left(x_{1}\right) w_{2}^{a_{2}}\left(x_{2}\right)} \exp \left(-\phi a_{1} a_{2}\left(\log \frac{w_{1}\left(x_{1}\right)}{w_{1}(l)}\right)\left(\log \frac{w_{2}\left(x_{2}\right)}{w_{2}(l)}\right)\right)
$$

which is also a model included in the type of the PDF given in (d) with parameters $a_{1}-1$ and $a_{2}-1$. Therefore, its hazard rate functions are

$$
h_{i}^{w}(s \mid t)=\left(a_{i}-1\right)\left(1-\phi^{\prime}\left(a_{3-i}-1\right) \log \frac{w_{3-i}(t)}{w_{3-i}(l)}\right) \frac{w_{i}^{\prime}(s)}{w_{i}(s)}
$$

for $i=1,2$. Hence (a) holds.

\section{Acknowledgement}

JN is partially supported by Ministerio de Ciencia y Tecnología and Fundación Séneca under grants MTM2009-08311 and 08627/PI/08. JN thanks the support of the Government of Kerala, India, under the ERUDITE scheme during his visit to Cochin University of Science and Technology. 


\section{References}

Arnold, B.C., Castillo, E., Sarabia, J.M., 1999. Conditional Specification of Statistical Models. Springer Verlag, New York.

Arnold, B.C., Strauss, D., 1988. Bivariate distributions with conditionals in prescribed exponential families. J. Royal Statist. Soc. Ser. B 53, 365-375.

Bartoszewicz, J., 2009. On a representation of weighted distributions. Statist. Probab. Lett. 79, 1690-1694.

Bartoszewicz, J., Skolimowska, M., 2006. Preservation of classes of life distributions and stochastic orders under weighting. Statist. Probab. Lett. 76, 587-596.

Blazej, P., 2008. Preservation of classes of life distributions under weighting with a general weight function. Statist. Probab. Lett. 78, 3056-3061.

Cox, D.R., 1959. The analysis of exponentially distributed lifetimes with two types of failure. J. Royal Statist. Soc. Ser. B 21, 411-421.

Di Crescenzo, A., Longobardi, M., 2004. A measure of discrimination between past lifetime distributions. Statist. Probab. Lett. 67, 173-182.

Ebrahimi, N., Kirmani, S.N.U.A., 1996. A characterization of the proportional hazards model through a measure of discrimination between two residual life distributions. Biometrika 83, 233-235.

Ebrahimi, N., Kirmani, S.N.U.A., Soofi, E.S., 2007. Multivariate dynamic information. J. Multiv. Anal. 98, 328-349

Ebrahimi, N., Soofi, E.S., Soyer, R., 2010. Information Measures in Perspective. Inter. Statist. Rev. 78, 383-412.

Kullback, S., Leibler, R.A., 1951. On information and sufficiency. Ann. Math. Statist. 22, $79-86$. 
Maya, S.S., Sunoj, S.M., 2008. Some generalized information measures in the context of weighted models. Statistica, anno LXVIII (1), 71-84.

Navarro, J., Ruiz, J.M., del Aguila, Y., 2006. Multivariate weighted distributions. Statistics 40, 51-54.

Navarro, J., Sarabia, J.M., 2010. Alternative definitions of bivariate equilibrium distributions. J. Statist. Plann. Infer. 140, 2046-2056.

Navarro, J., Sarabia, J.M., 2011. Reliability properties of bivariate conditional proportional hazard rates models. J. Multiv. Anal. (in press). Published online first, DOI: 10.1016/j.jmva.2011.03.009

Sankaran, P.G., Sreeja, V.N., 2007. Proportional hazard model for multivariate failure time data. Comm. Statist. Theory Meth. 36, 1627-1641.

Sunoj, S.M., Linu, M.N., 2011. Dynamic cumulative residual Renyi's entropy. Statistics (in press). Published online first, DOI: 10.1080/02331888.2010.494730. 